\title{
MULHER NO PERÍODO PÓS-REPRODUTIVO E HIV/AIDS: PERCEPÇÃO E AÇÕES SEGUNDO O MODELO DE CRENÇAS EM SAÚDE ${ }^{1}$
}

\author{
Neide de Souza Praça², Joyce de Oliveira Souza3 ${ }^{3}$ Daniela Angelo de Lima Rodrigues ${ }^{4}$
}

\footnotetext{
${ }^{1}$ Extraído do relatório de pesquisa - Mulheres com idade igual ou superior a 50 anos: percepção e ações diante da epidemia de aids, apresentado à Escola de Enfermagem da Universidade de São Paulo (USP), 2008.

${ }^{2}$ Livre-Docente. Professora Associada do Departamento de Enfermagem Materno-Infantil e Psiquiátrica da Escola de Enfermagem da USP. São Paulo, Brasil. E-mail: ndspraca@usp.br

${ }^{3}$ Bacharel em Enfermagem pela Escola de Enfermagem da USP. São Paulo, Brasil. E-mail: joycap@gmail.com

${ }^{4}$ Doutoranda do Programa de Pós-Graduação em Enfermagem da Escola de Enfermagem da USP. Docente do Curso de Enfermagem da Universidade Nove de Julho. São Paulo, Brasil. E-mail: daniangelo@usp.br
}

RESUMO: As evidências sugerem o crescente número de mulheres com idade igual ou superior a 50 anos com HIV/aids, no país. Este estudo descritivo, exploratório, realizado em 2008, teve como objetivo geral analisar a percepção sobre HIV/aids de mulheres, nesta faixa etária, frequentadoras de uma Unidade Básica de Saúde com Programa Saúde da Família do município de São Paulo. Foram entrevistadas 33 mulheres utilizando-se um formulário com questões estruturadas segundo os constructos do Modelo de Crenças em Saúde. Este, também norteou a análise dos dados. Verificou-se que os sujeitos se percebiam suscetíveis à infecção e reconheciam a severidade da aids, mas não transpunham barreiras para a prevenção da transmissão do HIV pela via sexual, embora orientassem familiares mais jovens sobre medidas preventivas. Conclui-se que, embora reconheçam a severidade da aids, as mulheres não transpõem barreiras para realizar ações de prevenção de infecção pelo HIV.

DESCRITORES: Saúde da mulher. Aids. Idoso. Enfermagem. Percepção.

\section{POST-REPRODUCTIVE WOMEN AND HIV/AIDS: PERCEPTIONS AND ACTIONS ACCORDING TO THE HEALTH BELIEF MODEL}

\begin{abstract}
Evidence reveals an increasing number of women aged 50 or over who have HIV/AIDS in Brazil. This descriptive and exploratory study, conducted in 2008, aimed to analyze perceptions about HIV/AIDS from women in this age group who attended a Basic Health Unit under the Family Health Program in São Paulo, Brazil. A structured-question questionnaire was used to interview 33 women according to the constructs of the Health Belief Model, which also guided data analysis. The data showed that these individuals were aware of their susceptibility to the infection and understood the severity of AIDS as well, but they did not break the obstacles for the prevention and transmission of HIV through sex. On the other hand, they have oriented their younger relatives about prevention measures. It has been concluded that, while recognizing the severity of AIDS, women do not transpose barriers in order to perform HIV infection prevention actions.
\end{abstract}

DESCRIPTORS: Women's health. AIDS. Aged. Nursing. Perception.

\section{LA MUJER EN EL PERÍODO POST-REPRODUCTIVO Y EL VIH/SIDA: PERCEPCIÓN Y ACCIONES SEGÚN EL MODELO DE CREENCIAS EN SALUD}

RESUMEN: Las evidencias sugieren un creciente número de mujeres con edad igual o superior a 50 años con VIH/SIDA en el país. El presente es un estudio descriptivo, exploratorio, realizado en 2008, con el objetivo general de analizar la percepción sobre el VIH/SIDA de mujeres en este grupo etario que asisten a una unidad de atención primaria del Programa de Salud Familiar, de la Municipalidad de Sao Paulo, Brazil. Para el estudio se entrevistaron 33 mujeres utilizando un formulario con preguntas estructuradas según las bases del Modelo de Creencias en Salud, el cual también norteó el análisis de los datos. Se verificó que los sujetos se percibían susceptibles a la infección y reconocían la severidad del SIDA, pero no traspasaban las barreras para la prevención de la transmisión del VIH por la vía sexual, a pesar de orientar a familiares más jóvenes sobre medidas preventivas. Se concluye que aunque reconocen la gravedad del SIDA, las mujeres no traspasan las barreras para llevar a cabo acciones para prevenir la infección por el VIH.

DESCRIPTORES: Salud de la mujer. SIDA. Anciano. Enfermería. Percepción. 


\section{INTRODUÇÃO}

Os idosos demonstram dificuldade para se perceber e agir enquanto atores no processo saúdedoença e para atribuir significado a essa fase de suas vidas. As principais causas dessa dificuldade relacionam-se às carências de informações sobre doenças de transmissão sexual, a aids em especial, e sobre as ações e percepções que direcionam seus comportamentos em saúde. ${ }^{1}$

São diversos os fatores que dificultam o uso de preservativos pelo casal cuja mulher está no período pós-reprodutivo: dificuldade de negociação entre os parceiros para adoção de práticas sexuais mais seguras, dúvidas quanto à efetividade do preservativo e ao seu uso, reduzido conhecimento sobre as vias de transmissão do HIV, e reduzida percepção de risco para a infecção pelo HIV motivada pela confiança da mulher no relacionamento estável e pela crença na imunidade à doença. Muitas vezes, o diálogo sobre aids com os familiares é dificultado pela falta de hábito de falar sobre sexualidade, o que concorre para o aumento de comportamentos de risco para a transmissão do HIV. Outro fator encontrado nos estudos reside na crença das mulheres desta faixa etária de que os jovens são mais suscetíveis à infecção, além de preferirem não abordar o tema aids, pois se vêem muito distantes da síndrome..$^{2-5}$

Diante desta situação faz-se necessário identificar, junto à mulher na fase pós-reprodutiva, quais são suas informações, crenças e ações diante da epidemia de aids. Dado ao amplo universo de contextos de vida dessas mulheres, e com a finalidade de contribuir para a construção de ações educativas voltadas a este segmento, julgou-se relevante realizar esta pesquisa, cuja motivação revela-se, também, na constatação de reduzido número de publicações com abordagem na temática voltada à mulher que vivencia esta fase do curso da vida, no País. Cabe esclarecer que se empregou o termo "mulher no período pós-reprodutivo" levando-se em consideração que aproximadamente $70 \%$ da amostra encontravam-se na faixa etária acima de 56 anos, portanto, vivendo a pós-menopausa, caracterizando o período para além do reprodutivo.

Diante do exposto, o presente estudo pretendeu responder as seguintes questões: como as mulheres no período pós-reprodutivo percebem a epidemia de aids? Elas realizam ações para evitar a transmissão do HIV? Para responder as questões, optou-se pela adoção do Modelo de Crenças em Saúde como norteador da coleta e da análise dos dados. Faz-se necessário, portanto, discorrer brevemente sobre o mesmo.

\section{Modelo de Crenças em Saúde}

A teoria que originou o Modelo de Crenças em Saúde considera a percepção do indivíduo sobre seu mundo como fator determinante de suas ações, e estas dependem de suas escolhas em dadas situações.

Os indivíduos realizam ações para desviar, proteger ou controlar condições de saúde-doença se eles se percebem como suscetíveis a essa condição. Também agirão se acreditarem que a condição de saúde tem potencialmente sérias conseqüências para sua vida. ${ }^{6}$

Para melhor explicitar o Modelo de Crenças em Saúde, apresentam-se seus componentes:5,7-10

Suscetibilidade percebida: refere-se a riscos subjetivos de contrair um agravo. É a percepção variável de indivíduo para indivíduo. Uma pessoa pode adotar medidas que evitam totalmente o agravo; outras, embora admitam a possibilidade de contrair uma doença, não acreditam que tal fato ocorra com elas. Por outro lado, há pessoas que fortemente se vêem em real perigo de contrair uma doença.

Severidade percebida: assim como a anterior, também é variável de indivíduo para indivíduo. O grau de severidade percebido depende do nível de estímulo emocional e da intensidade das perdas criadas pelo pensamento de uma doença e pelas dificuldades que o indivíduo percebe que uma dada condição de saúde criará para ele, como por exemplo a morte, a redução temporária ou permanente da capacidade física ou mental, dor e/ou implicações no trabalho, na vida familiar e nas relações sociais.

Relação benefícios e barreiras percebidos: dependerá de como o indivíduo vê a relação entre o custo e a ação de saúde. Caso os custos sejam superiores aos benefícios derivados da ação, os procedimentos para transpor as barreiras não se efetivarão. Dentre as barreiras incluem-se os custos financeiros, as reações fóbicas e físicas, os fatores de acessibilidade e as características de personalidade.

Motivação para agir: os fatores que influenciam a tomada de decisão do indivíduo para adotar medidas de prevenção em saúde dependem da combinação que a pessoa faz entre sua percepção de suscetibilidade e de severidade para uma dada doença e a avaliação entre as barreiras (aspectos negativos) e os benefícios que suas ações acarretarão. Se a motivação para desenvolver uma ação em saúde é alta e o indivíduo vê os aspectos negativos envolvidos como relativamente baixos, a ação em questão se efetiva; por outro lado, se ocorrer o inverso, os aspectos negativos funcionam como barreiras para evitar qualquer ação. Quando am- 
bos, motivação para desenvolver ação em saúde e barreiras contra a ação, estão fortemente presentes, há um conflito de difícil solução.

Portanto, esta pesquisa tem como objetivo geral analisar a percepção sobre HIV/aids de mulheres no período pós-reprodutivo, frequentadoras de uma Unidade Básica deSaúde (UBS) com Programa Saúde da Família (PSF) do Município de São Paulo.

Como objetivos específicos foram definidos: identificar a percepção de suscetibilidade à infecção pelo HIV pelas mulheres; descrever como percebem a severidade da aids; identificar barreiras percebidas para adesão a ações preventivas da infecção pelo HIV; e verificar se adotam ações para a prevenção da transmissão do HIV pela via sexual.

Diante do exposto, este estudo tem a finalidade de obter subsídios que contemplem ações de enfermagem voltadas à prevenção da infecção pelo HIV, para esta faixa etária.

\section{METODOLOGIA}

Trata-se de um estudo do tipo descritivo, exploratório, sobre aids, que teve como eixo norteador o Modelo de Crenças em Saúde.

Os dados foram coletados na UBS com PSF Jardim São Jorge, localizada na região oeste do município de São Paulo. A sua área de abrangência é constituída por prédios de um projeto habitacional para moradores de baixa renda, e por casas e barracos construídos ilegalmente.

A população correspondeu a mulheres com idade igual ou superior a 50 anos, usuárias da UBS, campo do estudo. A amostra por conveniência foi composta por 33 mulheres, que atenderam os critérios de inclusão definidos conforme segue: ter idade igual ou superior a 50 anos; estar cadastrada no PSF; e concordar em participar da pesquisa.

Vale esclarecer que o tamanho da amostra limitou-se às 33 mulheres, pois houve necessidade de alterar o meio de contato com as potenciais entrevistadas. A princípio, estas seriam contatadas durante atividade recreativa na unidade de saúde, porém mudanças na estrutura do serviço impossibilitaram este contato, obrigando as autoras a buscar as potenciais entrevistadas em seu domicílio, o que prejudicou o alcance de amostra de maior amplitude.

A coleta de dados ocorreu no período de maio a julho de 2008 e se iniciou após aprovação pelo Comitê de Ética em Pesquisa da Secretaria de Saúde do Município de São Paulo, sob o n ${ }^{\circ}$ 109/08 - CEP/SMS. CAAE: 0048.162.000-08, e após a obtenção de autorização do diretor da unidade campo do estudo.
No período definido para a coleta de dados, as participantes foram identificadas dentre as mulheres cadastradas na unidade, e contatadas após sorteio segundo a ordem de matrícula na mesma. Uma vez identificada cada potencial participante, a pesquisadora, em companhia de agentes de saúde, realizava visita domiciliar à mulher, momento em que a informava sobre os objetivos e a proposta do estudo, buscando sua concordância em participar do mesmo. Após a aceitação era realizada a entrevista individual, utilizando-se um formulário estruturado com 45 questões fechadas distribuídas conforme os componentes do Modelo de Crenças em Saúde, e caracterizadas segundo os seguintes temas: dados sociodemográficos, informações sobre aids, percepção de suscetibilidade à infecção pelo HIV, percepção de severidade da aids, relação benefícios e barreiras percebidos, e motivação para agir em direção à prevenção da infecção.

O instrumento de coleta de dados se constituiu na replicação de formulário aplicado em estudo anterior, realizado com mulheres na mesma faixa etária, frequentadoras de atividades recreativas em unidade de apoio social localizada na região leste do município de São Paulo. ${ }^{11} \mathrm{O}$ formulário matriz, ligeiramente modificado, foi elaborado com base em resultados de estudos sobre a temática aids e a mulher idosa, realizados em comunidades de baixa renda do município de São Paulo. ${ }^{3,5}$ As respostas de suas questões com foco na epidemia de aids foram assinaladas como Falsas ou Verdadeiras, conforme as informações, a percepção, e as ações das mulheres entrevistadas.

Os dados foram lançados em banco de dados confeccionado no programa Excel ${ }^{\circledR}$ e os resultados são apresentados por meio de números absolutos e percentuais, com análise descritiva.

O Termo de Consentimento Livre e Esclarecido, elaborado em duas vias, foi apresentado aos sujeitos que, após concordarem, o assinaram e receberam uma de suas cópias; a outra cópia permaneceu em poder da pesquisadora. A entrevista foi realizada de modo a garantir a privacidade da mulher que obteve garantia de não sofrer prejuízo no atendimento na UBS/PSF caso desistisse ou se recusasse a participar da pesquisa.

\section{RESULTADOS E DISCUSSÃO}

\section{Caracterização da amostra}

À Tabela 1 tem-se a caracterização sociodemográfica da amostra. Vale acrescentar que dados como escolaridade e estado civil concordam com 
estudo realizado em Santa Catarina, com indivíduos de ambos os sexos, com HIV/aids. ${ }^{12}$ Ambos refletem as mudanças no perfil da epidemia ao longo de sua trajetória, no país.

Tabela 1 - Características sóciodemográficas da amostra. São Paulo-SP, 2008

\begin{tabular}{|c|c|c|}
\hline Variável & $\mathbf{n}$ & $\%$ \\
\hline \multicolumn{3}{|l|}{ Idade (anos) } \\
\hline $51-55$ & 10 & 30,4 \\
\hline $56-60$ & 8 & 24,2 \\
\hline $61-65$ & 6 & 18,2 \\
\hline $66-70$ & 5 & 15,1 \\
\hline $71-75$ & 4 & 12,1 \\
\hline \multicolumn{3}{|l|}{ Escolaridade } \\
\hline Analfabeta & 3 & 9,1 \\
\hline Ensino fundamental $-1^{a}$. a $4^{a}$. série & 26 & 78,7 \\
\hline Ensino fundamental - $5^{a}$. a $8^{a}$. série & 3 & 9,1 \\
\hline Ensino médio completo & 1 & 3,1 \\
\hline \multicolumn{3}{|l|}{ Estado civil } \\
\hline Casada & 16 & 48,4 \\
\hline Viúva & 10 & 30,4 \\
\hline Divorciada & 4 & 12,1 \\
\hline Desquitada & 2 & 6,1 \\
\hline Solteira & 1 & 3,1 \\
\hline \multicolumn{3}{|l|}{ Mora com companheiro } \\
\hline Sim & 18 & 54,5 \\
\hline Não & 15 & 45,5 \\
\hline \multicolumn{3}{|l|}{ Número de parceiros no último ano } \\
\hline 1 & 18 & 54,5 \\
\hline 2 & 5 & 15,1 \\
\hline Não teve & 10 & 30,4 \\
\hline \multicolumn{3}{|l|}{ Número de parceiros na vida } \\
\hline Nenhum & 1 & 3,1 \\
\hline 1 & 23 & 69,6 \\
\hline 2 & 5 & 15,1 \\
\hline 3 a 5 & 2 & 6,1 \\
\hline Mais de 5 & 2 & 6,1 \\
\hline \multicolumn{3}{|l|}{ Tem namorado } \\
\hline Sim & 4 & 26,6 \\
\hline Não & 6 & 40,0 \\
\hline Não se aplica & 5 & 33,3 \\
\hline \multicolumn{3}{|l|}{ Renda familiar (em salários mínimo)* } \\
\hline Não sabe & 2 & 6,1 \\
\hline 1 a 2,5 & 26 & 78,7 \\
\hline 3 a 5 & 2 & 6,1 \\
\hline Mais de 5 & 3 & 9,1 \\
\hline \multicolumn{3}{|l|}{ Ocupação } \\
\hline Do lar & 20 & 60,6 \\
\hline Trabalho remunerado & 9 & 27,3 \\
\hline Aposentada & 4 & 12,1 \\
\hline \multicolumn{3}{|l|}{ Religião } \\
\hline Católica & 23 & 69,6 \\
\hline Evangélica & 9 & 27,3 \\
\hline Espírita & 1 & 3,1 \\
\hline Total & 33 & 100,0 \\
\hline
\end{tabular}

* Salário Mínimo vigente à época R\$ 400,00.

\section{Informações sobre aids}

Ao serem apresentadas às questões do instrumento de coleta de dados, as mulheres indicaram o significado que as afirmativas tinham para elas, apontando se as consideravam falsas ou verdadeiras. Os dados obtidos são apresentados à Tabela 2, a seguir.

Tabela 2 - Conhecimento dos sujeitos sobre HIV/ aids. São Paulo-SP, 2008

\begin{tabular}{lrc}
\hline Variável & $\mathbf{n}$ & $\%$ \\
\hline HIV é o nome dado à aids & 4 & 12,1 \\
Falso & 25 & 75,8 \\
Verdadeiro & 4 & 12,
\end{tabular}

Indivíduos que devem fazer teste anti-HIV

Homem com múltiplos parceiros

$$
\text { Falso }
$$

Verdadeiro

33100

Homem que faz sexo com outros homens Falso Verdadeiro

Usuário de drogas injetáveis

Falso

Verdadeiro

33100

Mulher com múltiplos parceiros

Falso

Verdadeiro

$33^{-} \quad 100$

Homem com relacionamento fora do casamento

Falso

Verdadeiro

Mulher com relacionamento fora do casamento

Falso

Verdadeiro

Casal que não utiliza preservativo

Falso

Verdadeiro

$4 \quad 12,1$

Gestante

Falso

Verdadeiro

$29 \quad 87,9$

Adolescente sem parceiro fixo Falso Verdadeiro

$33 \quad 100$

este anti-HIV é obrigatório no pré-natal

Falso

Verdadeiro

$2 \quad 6,1$

3193,9

Ser infectado pelo HIV é o mesmo que ter aids

Falso

Verdadeiro

$2 \quad 6,1$

3193,9

Não sabe

Existe vacina contra aids

Falso

Verdadeiro

Não sabe

$3 \quad 9,1$

$30 \quad 90,9$

$7 \quad 21,3$

2369,6

$3 \quad 9,1$

Total 
À Tabela 2 verifica-se que as mulheres possuíam informações adequadas sobre as situações de risco para a infecção pelo HIV, bem como sobre os indivíduos que devem se submeter à testagem anti-HIV. Sob a luz do Modelo de Crenças em Saúde, esta situação deveria realçar a percepção de sua suscetibilidade diante da possibilidade de infecção pelo HIV, motivando-as para adotar ações de prevenção, porém, esta não foi uma condição predominante verificada nos dados obtidos, e apresentados adiante.

Os achados concordaram com estudo de base populacional, referente ao ano de 2005, que mostrou que $54 \%$ das mulheres entre 16 e 65 anos de idade, no país, apresentavam informações sobre $\mathrm{HIV} /$ aids, porém não se percebiam em risco de infecção, enquanto que 51\% tinham informação e alguma percepção de risco. O estudo mostrou, também, que no período entre 1998 e 2005, houve aumento na proporção dos indivíduos que referiram não ter risco frente à aids, apesar do aumento do nível de conhecimento em geral. ${ }^{13}$

Ainda que demonstrassem reter informações sobre situações de risco para a transmissão do HIV, a recomendação de testagem para as pessoas que a amostra considerava como suscetíveis ao vírus da aids, bem como para a gestante, indicou que reconheciam a triagem como caminho para identificar a suscetibilidade à infecção.

Por desconhecer o significado de termos básicos empregados na epidemia de aids, considerando semelhantes o doente e o infectado pelo HIV, tal condição pode ter prejudicado a percepção das mulheres quanto à severidade da aids, apresentada adiante, pois, podem ter uma visão distorcida sobre a progressão da trajetória individual da doença e, com isso, considerá-la com maior ou com menor gravidade, o que poderá repercutir no seu cotidiano.

\section{Percepção de suscetibilidade}

Ao se considerar a percepção de suscetibilidade à infecção pelo HIV, verificou-se que $72,7 \%$ da amostra identificaram suscetibilidade ao vírus da aids diante da presença de Doenças Sexualmente Transmissíveis, associando-as à transmissão do HIV. Outro fator de suscetibilidade percebido residiu na associação que fizeram entre HIV e relação sexual desprotegida com parceiro soropositivo ao vírus $(63,2 \%)$, fato que se confirma quando associaram a prática sexual desprotegida à infecção $(96,9 \%)$, e à afirmação de que a atividade sexual as tornaria suscetíveis ao vírus $(81,8 \%)$. O preservativo como meio de evitar a transmissão do HIV foi verificado junto a $87,8 \%$ das respondentes fato que as levou a perceber sua suscetibilidade à infecção diante de relações sexuais sem uso de preservativos $(100 \%)$.

A mulher com mais de 60 anos e ou que vivencia a menopausa e ou que se submeteu à laqueadura tubárea foi identificada como suscetível à infecção pelo HIV, desde que não adira aos preservativos, nas seguintes proporções: 90,9\%, $84,9 \%$ e $81,8 \%$, respectivamente.

Outras possibilidades percebidas que tornam as mulheres suscetíveis ao vírus da aids, apontadas pela amostra, relacionaram-se à vida social e à busca por tratamento, pois 93,9\% apontaram consultórios médicos, dentários ou hospitais como fontes de infecção pelo HIV, portanto, frequentálos as tornaria suscetíveis à infecção; o mesmo foi verificado para o uso de objetos em farmácias e em salões de cabeleireiro, ambos apontados por $87,9 \%$ das mulheres.

Vale destacar que a totalidade das mulheres se percebia suscetível à infecção diante de hemotransfusões e que $72,7 \%$ não consideravam que seus parceiros atuais ou anteriores pudessem infectar-se com o vírus da aids.

Os achados confirmaram a reduzida percepção da própria suscetibilidade, dado, também, demonstrado em estudo realizado com 14 homens e oito mulheres maiores de 50 anos, soropositivos para o HIV, residentes em dois municípios de Santa Catarina, que mostrou os motivos que levaram estes sujeitos a se submeter à testagem anti-HIV e que confirmaram sua suscetibilidade ao vírus da aids: relações sexuais sem uso de preservativo, presença de doenças oportunistas, uso de droga injetável, descoberta de soropositividade do parceiro e outras doenças. Vale acrescentar que nenhum sujeito referiu uso de preservativo anteriormente à descoberta da própria infecção. Os dados mostraram que a relação sexual foi a principal via de infecção, e que a falta de adesão ao preservativo foi o fator que pode ter facilitado a infecção. ${ }^{12}$

Embora percebendo a suscetibilidade à infecção pelo HIV, a maioria da amostra referiu que não se previne da infecção, ainda que reconhecesse que a idade avançada, a menopausa e a laqueadura tubárea sejam fatores que predispõem a infecção na mulher, caso não adira ao preservativo. Por outro lado, chama atenção o elevado número de entrevistadas que se percebiam suscetíveis ao 
HIV ao se submeter a hemotransfusões, e a procedimentos em hospitais e consultórios médicos e dentários, assim como em farmácias e salões de beleza. Dados, também, encontrados em outros estudos com populações semelhantes. ${ }^{3,5}$

\section{Percepção de severidade da aids}

A severidade percebida para a aids ganhou destaque, na amostra, diante da afirmação de que a síndrome é incurável $(75,7 \%)$, porém, esta condição pode ser atenuada ao considerarem que o indivíduo acometido pelo agravo tem maior sobrevida e tem disposição para o trabalho. $\mathrm{Na}$ perspectiva do Modelo de Crenças em Saúde, ambas as condições se sobrepõem, reduzindo a amplitude da ausência de cura, pelo prolongamento da vida dos infectados que, submetidos ao tratamento antiretroviral, têm a possibilidade de realizar atividades econômicas e sociais.

A letalidade da aids foi um dos temas de estudo epidemiológico, no qual foram pesquisados os casos de aids em população de Fortaleza-CE, com idade igual ou superior a 50 anos, notificados ao Ministério da Saúde. Os achados mostraram que, no período entre 1991 e 2001, o coeficiente de letalidade foi maior para a faixa etária de 55 a 59 anos, e na comparação entre os sexos, a mulher apresentou menor coeficiente de letalidade que o homem, $29,6 \%$ e $46,5 \%$, respectivamente. A conclusão dessa pesquisa relacionou a causa de aumento dos casos de aids na população sob estudo à expansão do número de relações sexuais, às novas terapias antiretrovirais e ao aumento da sobrevida dos doentes de aids. ${ }^{14}$

\section{Benefícios e barreiras percebidos}

Sob esta perspectiva, as mulheres pontuaram situações consideradas como barreiras diante da infecção pelo HIV/aids, dentre elas destaca-se a dificuldade de o parceiro concordar com o uso do preservativo (87,8\%). Igual percentual mostrou que a amostra acreditava que em caso de recusa, ela deveria insistir no uso do preservativo, porém, $66,7 \%$ percebiam a suscetibilidade da mulher diante da possibilidade de agressão. Paralelamente, $60,6 \%$ sentiam dificuldade para negociar com o parceiro o uso do preservativo. Esta condição mostrou que a percepção de fatores de suscetibilidade, pela amostra, não foi suficiente para influenciá-la na tomada de decisão diante da comparação entre as barreiras que terá de enfrentar e os benefícios obtidos com a prevenção da infecção.

\section{Ações para prevenção da infecção pelo HIV/ aids}

As mulheres agem após avaliar barreiras e benefícios que terão ao transpô-las. Quando solicitadas a informar as próprias ações de prevenção à infecção pelo HIV citaram não usar objetos de uso por desconhecidos $(87,9 \%)$, não usar drogas injetáveis $(75,7 \%)$ e não ser parceira de usuário de drogas injetáveis $(81,8 \%)$.

Chama atenção que $69 \%$ das mulheres referiram preocupação com filhos e netos, quando o assunto é HIV/aids.

Vê-se que as ações desencadeadas pelas mulheres, diante da epidemia, e de sua percepção sobre a mesma, as direcionaram para a preocupação com filhos e netos. Achados concordantes foram verificados em estudos realizados com populações semelhantes, onde as mulheres, na mesma faixa etária de interesse, preocupavam-se em orientar os descendentes sobre medidas de prevenção à infecção pelo HIV, principalmente para os do sexo masculino, que consideravam com comportamentos de maior risco de infecção pela via sexual. ${ }^{3,5}$

Perceber a severidade da aids e reconhecer situações de suscetibilidade à infecção pelo HIV deveriam gerar comportamentos preventivos da transmissão deste vírus pela via sexual, porém, a maior preocupação da amostra residiu na prevenção diante de tratamentos em instituições de saúde e ou no cuidado estético (salões de beleza). Essa condição mostrou a reduzida percepção de suscetibilidade das mulheres entrevistadas ao avaliar a transmissibilidade e a via sexual de transmissão do vírus da aids. Estes dados são concordes com resultados obtidos em estudos que mostraram a reduzida preocupação da mulher na faixa etária de interesse em se prevenir da transmissão do HIV por via sexual. 3,5

Nesse aspecto, as mulheres na faixa etária de interesse, participantes deste estudo, demonstraram reduzida preocupação, situação que se agrava quando se avaliam os casos de aids notificados ao Sistema de Informações em Saúde do DATASUS, no período de 1995 a 2005, onde a categoria de exposição com maior representatividade foi decorrência de relação sexual heterossexual $(51,6 \%) \cdot{ }^{15}$

Diante dos resultados obtidos, verificou-se que os achados concordam com aqueles obtidos em estudos realizados, no município de São Paulo, com sujeitos em situação de vida semelhante. ${ }^{3,5}$ 


\section{CONCLUSÕES}

Este estudo, norteado pelo Modelo de Crenças em Saúde possibilitou verificar que as mulheres no período pós-reprodutivo, de baixa renda e com reduzida escolaridade, frequentadoras de uma UBS com PSF no município de São Paulo, retém informações sobre a epidemia de aids, reconhecem a severidade do agravo, assim como os fatores que as tornam suscetíveis à infecção, porém não transpõem barreiras para realizar ações de prevenção, embora evitem expor-se quando utilizam instituições que cuidam de saúde e de estética. Estas afirmações embasam-se nos dados obtidos, quais sejam: possuem informações adequadas sobre a aids e percebem sua suscetibilidade ao $\mathrm{HIV}$, relacionando-o à presença de DST e à relação desprotegida com parceiro infectado; a maioria vê consultórios, salões de beleza e farmácias como fontes de infecção; e identifica a síndrome como incurável, porém sua dificuldade para convencer o parceiro para adesão ao preservativo constitui-se em barreira que prejudica a realização de ações de prevenção da infecção. Estas estão voltadas essencialmente para a orientação de filhos e netos no esforço para a prevenção da transmissão do HIV.

Ainda que os resultados se limitem aos sujeitos do estudo, os achados devem servir de alerta para os profissionais da área da saúde, cuidadores deste segmento populacional, no sentido de reconhecer as necessidades deste grupo etário e identificar seus membros como partícipes no processo saúde-doença, motivando-os à incorporação das informações e das percepções que possuem rumo à modificação de comportamentos para a prevenção da infecção pelo vírus da aids.

As limitações deste estudo relacionam-se ao número de sujeitos, e à sua localização geográfica - moradores de uma dada comunidade -, o que impede a generalização dos achados, porém estes são considerados válidos, pois refletem condições semelhantes verificadas em pesquisas de maior abrangência, realizadas em outras regiões do país.

Sugere-se, portanto, que o profissional de saúde seja o facilitador das mudanças de comportamentos para a prevenção da transmissão do HIV, pela mulher que vivencia o período pósreprodutivo.

\section{Agradecimentos}

As autoras agradecem ao CNPq pela Bolsa de Iniciação Científica PIBIC/USP/CNPq que possibilitou a realização deste estudo.

\section{REFERÊNCIAS}

1. UchôaE,FirmoJOA, Lima-CostaMFF.Envelhecimento e saúde: experiência e construção cultural. In: Minayo MCS, Coimbra Junior CEA organizadores. Antropologia, saúde e envelhecimento. Rio de Janeiro (RS): Fiocruz; 2004. p.25-35.

2. Finkler L, Braga P, Gomes WB. Percepção de casais heterossexuais em relação à suscetibilidade de infecção por HIV/aids. Interação. 2004; 8(1):113-22.

3. Lima DA. A mulher com idade igual ou superior a 50 anos e a epidemia de aids: percepção e ações de moradoras de uma comunidade de baixa renda [dissertação]. São Paulo (SP): Universidade de São Paulo, Escola de Enfermagem; 2006.

4. Oltramari LC, Otto LS. Conjugalidade e aids: um estudo sobre infecção entre casais. Psicologia Social 2006 Ago; 18(3):55-61.

5. Praça NS, Barbosa FOS. HIV/aids e a mulher com idade igual ou superior a 50 anos. In: Paula CC, Padoin SMM, Schaurich D organizadores. Aids: o que ainda há para ser dito? Santa Maria (RS): Editora UFSM; 2007. p.157-68.

6. Rosenstock IM, Strecher VJ, Becker MH. The Health Belief Model and HIV risk behavior change. In: DiClemente RJ, Peterson JL. Preventing AIDS: Theories and methods of behavioral interventions. New York (US): Plenum Press; 1994. p.5-24.

7. Rosenstock IM. Historical origins of the Health Belief Model. Health Educ Monogr. 1974 Out; 20(4):328-35

8. Strecher VJ, Rosenstock IM. The Health Belief Model. In: Glanz K, Lewis FM, Rimer BK. Health Behavior and health education: theory, research and practice. $2^{\mathrm{a}}$ ed. San Francisco (US): Jossey-Bass; 1997. p.41-59.

9. Praça NS, Gualda DMR. Percepção de risco para $\mathrm{HIV}$ / aids de mulheres faveladas segundo o Modelo de Crenças em Saúde. Rev Esc Enf USP. 2001 Mar; 35(1):54-9.

10. Dela Coleta MF organizadora. Modelos para pesquisa e modificação de comportamentos em saúde. Taubaté (SP): Cabral; 2004.

11. Uehara J, Praça NS. Jogo educativo sobre HIV/aids para mulheres idosas. In: Anais do $16^{\circ}$. Simpósio Internacional de Iniciação Científica da USP (SIICUSP), 2008 Nov 5; Ribeirão Preto (SP): USP; 2008. CD-ROM.

12. Bertoncini BZ, Moraes KS, Kulkamp IC. Comportamento sexual em adultos maiores de 50 anos infectados pelo HIV. DST - J Bras Doenças Sex Transm. 2007 Abr; 19(2):75-9.

13. Ferreira MP. Nível de conhecimento e percepção de risco da população brasileira sobre o HIV/aids, 1998 e 2005. Rev Saúde Pública. 2008 Jun; 42 (supl $1): 65-71$. 
14. Feitoza AR, Souza AR, Araújo MFM. A magnitude da infecção pelo HIV-Aids em maiores de 50 anos no município de Fortaleza - CE. DST - J Bras Doenças Sex Transm. 2004 Out; 16(4):32-7.
15. Godoy VS, Ferreira MD, Silva EC, Gir E, Canini SRMS. O perfil epidemiológico da Aids em idosos utilizando sistemas de informações em saúde do DATASUS: realidades e desafios. DST - J Bras Doenças Sex Transm. 2008 Jan; 20(1):7-11. 